\title{
Actinomicosis de Pared Abdominal con Infiltración Hepática Simulando una Neoplasia Maligna. Reporte de un Caso
}

\author{
Abdominal Wall Actinomycosis with Hepatic Infiltration \\ Mimicking a Malignant Neoplasm. Case Report
}

Carlos Manterola ${ }^{1,2}$; Luis Grande ${ }^{3} \&$ Tamara Otzen $^{2}$

MANTEROLA, C.; GRANDE, L. \& OTZEN, T. Actinomicosis de pared abdominal con infiltración hepática simulando una neoplasia maligna. Reporte de un caso. Int. J. Morphol., 37(3):1033-1037, 2019.

RESUMEN: La infección por Actinomices (actinomicosis), es una entidad poco frecuente y que puede crear dificultades diagnósticas y terapéuticas; principalmente cuando por su presentación se asemeja a neoplasias malignas. El objetivo de este estudio fue reportar un caso de actinomicosis de pared abdominal con infiltración hepática y revisar la evidencia existente. Se trata de una paciente sexo femenino, de 33 años de edad, sin antecedentes quirúrgicos ni de utilización de dispositivos intra-uterinos. Consultó por dolor abdominal y masa palpable a nivel epigástrico. Se estudió con imágenes, las que permitieron verificar una masa de pared abdominal con trayecto fistuloso al hígado. Se realizó una exéresis amplia de la lesión antes descrita. Una vez extirpado el espécimen, se fue a estudio histopatológico, que reveló gránulos de azufre consistentes con actinomices. La paciente evolució de forma satisfactoria, sin inconvenientes. Presentamos un caso poco común de actinomicosis de pared abdominal con infiltración hepática. Cuando se encuentra una gran masa intraperitoneal, la actinomicosis debe incluirse en el proceso de diagnóstico diferencial.

PALABRAS CLAVE: Actinomicosis; Infección por Actinomices; Tumor abdominal; Masa abdominal; Absceso hepático.

\section{INTRODUCCIÓN}

Los Actinomices son bacilos Gram positivos anaerobios o microaerofílicos, que se caracterizan por desarrollar colonias de tipo micelial y presentar ramificaciones. Suelen ser de crecimiento lento. El género está conformado por 12 especies, entre las cuales, el más patógeno para el hombre es el Actinomyces israelii; sin embargo, existen otras especies patógenas para la especie humana son A. Bovis, A. Ericksonii, A. Naeslundii, A. Viscosus y A. Odontolyticus (Hamid et al., 2000). Es un saprófito habitual de las vías respiratorias, cavidad bucal, orofaringe, tracto gastrointestinal y genital femenino; por lo que es a partir de estas localizaciones desde donde suelen producirse la mayor parte de las infecciones reportadas. Es así como se estima que las localizaciones más comunes son cara y cuello (50 \% a $65 \%$ ), abdomen (20 $\%$ ) y tórax (15\%) (Boyanova et al., 2015).

Las infecciones causadas por Actinomices son infrecuentes; por lo que su diagnóstico complejo, ya que a la baja prevalencia, se ha de sumar el hecho que las mani- festaciones clínicas son atípicas; en especial, en aquellos casos en los que se presenta como lesión tumoral, infiltrando tejidos blandos u órganos adyacentes. Se caracteriza por ser una enfermedad crónica, supurativa, granulomatosa, pero no contagiosa (Yi et al., 2008).

A pesar que el $20 \%$ de las infecciones por Actinomices ocurren en la cavidad abdominal; la afectación de la pared abdominal es extremadamente poco frecuente (García García et al., 2001). Hasta la fecha, sólo se han reportado alrededor de 70 casos en la literatura entre adultos y niños (Filipovic et al., 2005; Kim et al., 2015; Wacharachaisurapol et al., 2017; Tsujimura et al., 2018). Los primeros reportes de esta entidad corresponden a Bradshaw en 1846 y Good en 1931 (Pujol Molinara et al., 2000; García García et al.).

En la mayor parte de los casos, los pacientes tienen el antecedente de cirugías previas por perforación de vísce-

\footnotetext{
${ }^{1}$ Departamento de Cirugía, Universidad de La Frontera, Temuco, Chile.

${ }^{2}$ Centro de Excelencia en estudios Morfológicos y Quirúrgicos (CEMyQ), Universidad de La Frontera, Temuco, Chile.

${ }^{3}$ Institut Hospital del Mar d'Investigacions Mèdiques (IMIM), Barcelona, España.

Financiado parcialmente por Proyecto MEC 80170022
} 
ra hueca. Por ejemplo, apendicitis aguda perforada, la que se asocia al $65 \%$ de los casos de actinomicosis abdominal (Deshmukh \& Heaney, 1986). Una vez ocurrida la perforación, la infección se disemina localmente de forma indolente, pudiendo tomar meses e incluso años antes de la manifestación de los síntomas (Meyer et al., 2000). Del mismo modo, se ha documentado la posibilidad de diseminación hepática y pulmonar vía hematógena; la que se puede asociar con la producción de nódulos que se pueden confundir con neoplasias malignas (Apotheloz \& Regamey, 1996; Yi et al.).

Los síntomas suelen ser inespecíficos, como fiebre, baja de peso y alteración de los hábitos intestinales, los que pueden atribuirse erróneamente a una enfermedad maligna (Apotheloz \& Regamey; Yi et al.).

El objetivo de este estudio fue reportar un caso de actinomicosis de la pared abdominal con infiltración hepática y revisar la evidencia existente.

\section{CASO CLÍNICO}

Presentamos el caso de una paciente de sexo femenino, 48 años de edad, que consultó por dolor abdominal localizado en cuadrante superior derecho, compromiso del estado general y sensación febril no cuantificada. Como antecedentes mórbidos destacaba hipotiroidismo en tratamiento con Eutirox 50 mg por día. No refería antecedentes quirúrgicos, ni hábitos tóxicos.

La exploración física era normal, con excepción de dolor palpatorio en epigastrio e hipocondrio derecho. No se palpaban masas ni vísceras.

Se solicitaron pruebas de laboratorio habitual e imágenes.

Las pruebas de laboratorio fueron normales, con excepción de una leucocitosis de 12.350 / $\mu \mathrm{L}$ y una VHS de 25 $\mathrm{mm}$.

La tomografía axial computada y la resonancia nuclear magnética permitieron verificar una masa de $66 \mathrm{~mm}$ de diámetro mayor, localizada en la unión de los segmentos IVa y II del hígado, comprometiendo el ligamento falciforme, con extensión hacia la pared abdominal y costal inferior derecha (Fig. 1).

Dadas las características de la lesión, con sospecha de neoplasia maligna, se programó para cirugía electiva, la que se realizó vía laparotómica el 24 de septiembre de 2018.
La paciente se hospitalizó el mismo día de la intervención. Se le realizaron las pruebas de laboratorio preoperatorias de rigor, que se encontraban dentro de límites normales. Se administró profilaxis antibiótica con Ceftriaxona $1 \mathrm{~g}$ y Metronidazol 500 mg vía intravenosa.

Una vez en quirófano y tras ser sometida a anestesia general inhalatoria, se procedió a la apertura de la cavidad abdominal mediante una laparotomía en J (Manterola, 2014); objetivándose una masa de aprox. 10 $\mathrm{cm}$ de diámetro, de aspecto inflamatorio, mal delimitada, que comprometía la pared abdominal y costal inferior derecha, extendiéndose mediante un trayecto fistuloso (del cual emanaba pus inodora), hasta la lesión hepática antes descrita. Se realizó disección y exéresis de la masa de la pared abdominal en bloque; posterior a lo cual, se movilizó el hígado seccionando sus elementos de sostén (Manterola et al., 2017); para proceder a la exéresis total de la lesión, con márgenes de seguridad. Finalmente, se decidió realizar colecistectomía por verificarse la co-existencia de colesterolosis de la vesícula biliar. El área cruenta del hígado, se cubrió con un parche pediculado de omento mayor; y el defecto de la pared, se reparó por planos, con material de sutura absorbible.

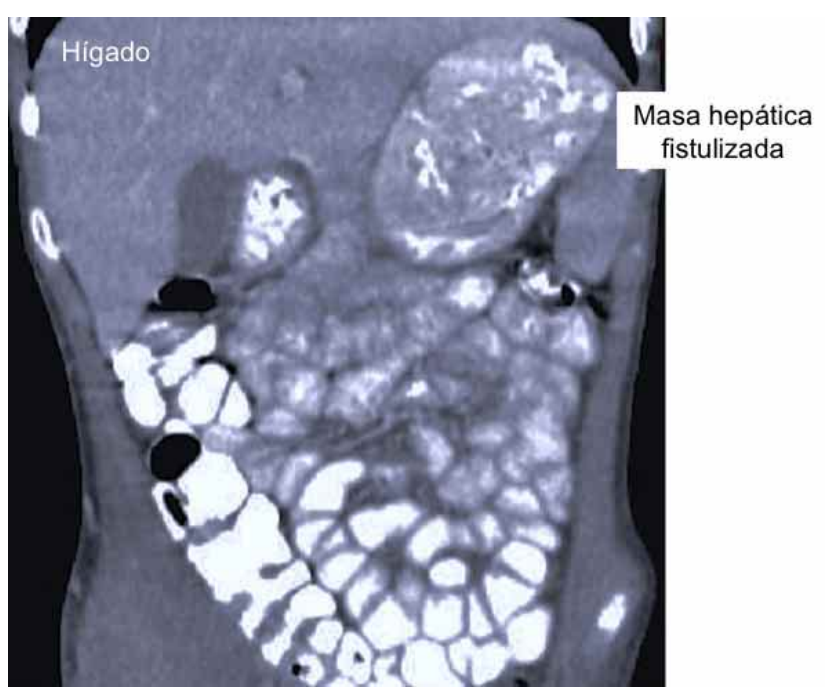

Fig. 1. Se aprecia masa hepática íntimamente adherida a la pared abdominal anterior.

La paciente evolucionó de forma adecuada, sin complicaciones; razón por la que fue dado de alta a los 3 días de la intervención, sin inconvenientes.

El informe del estudio histológico fue de colesterolosis vesicular. Tejido hepático y pared abdominal con proceso inflamatorio agudo y crónico, supurado, en granulación y organización fibrosa avanzada y adherencias 
en la periferia hepática, y la fibrosis de partes blandas adyacentes (Fig. 2). Se constató además, que el centro de la lesión presentaba exudado y un cúmulo de elementos filamentosos sugerentes de actinomices (Fig. 3). Los mismos elementos filamentosos dieron positivos con tinción PAS (Fig. 4A) y negativos con tinción de Grocott (Fig. 4B). Finalmente, la tinción de Gram, permitió confirmar el diagnóstico (Fig. 5).

Una vez certificado el diagnóstico, se indicó tratamiento con Amoxicilina a dosis de $1 \mathrm{~g}$ c/8 horas por 4 semanas.

En la actualidad, el paciente lleva 9 meses de seguimiento clínico y con imágenes, sin evidencias de recidiva.

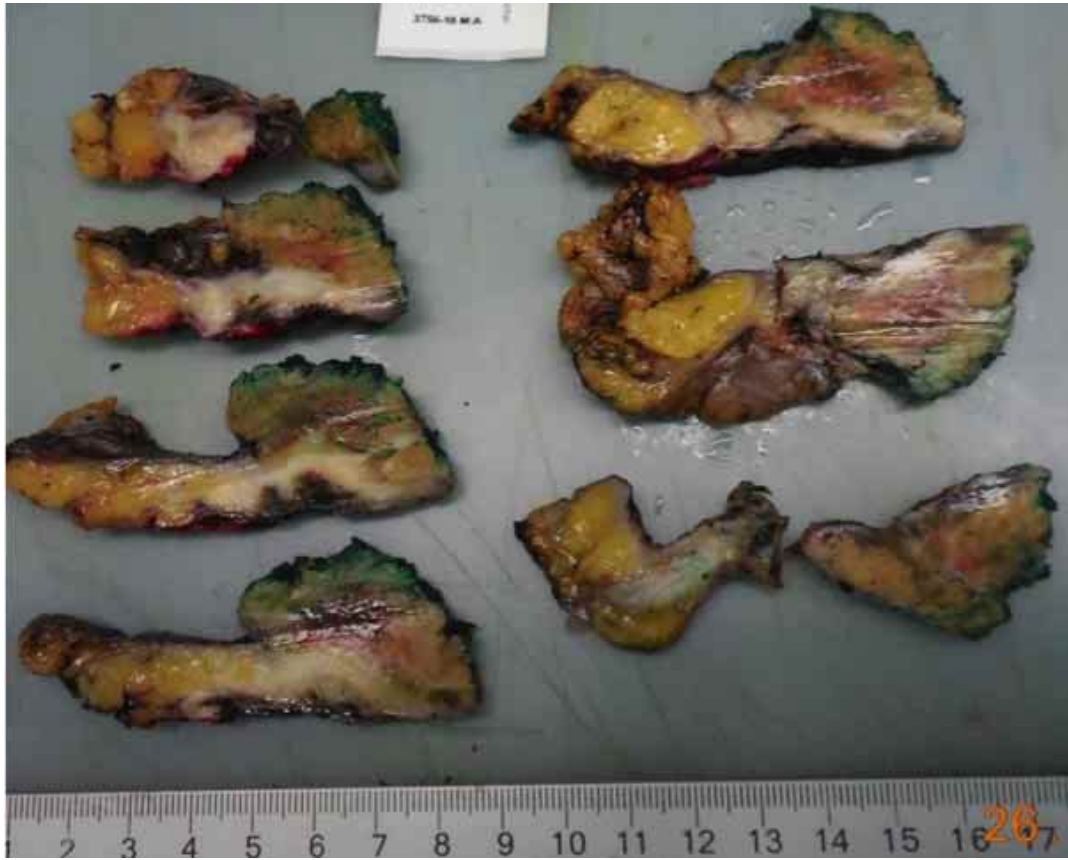

Fig. 2. Espécimen de masa abdominal y hepática resecada en bloque y fijada. Se puede apreciar la intensa fibrosis y adherencias en la periferia hepática, y la fibrosis de partes blandas adyacentes.

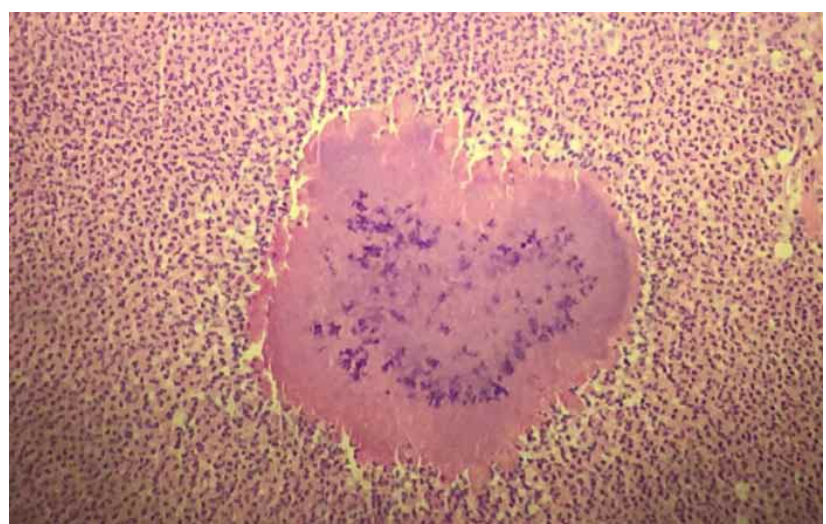

Fig. 3. Corte histológico de la pieza quirúrgica teñido con H\&E. Se aprecia en el centro del exudado, cúmulo de elementos filamentosos.

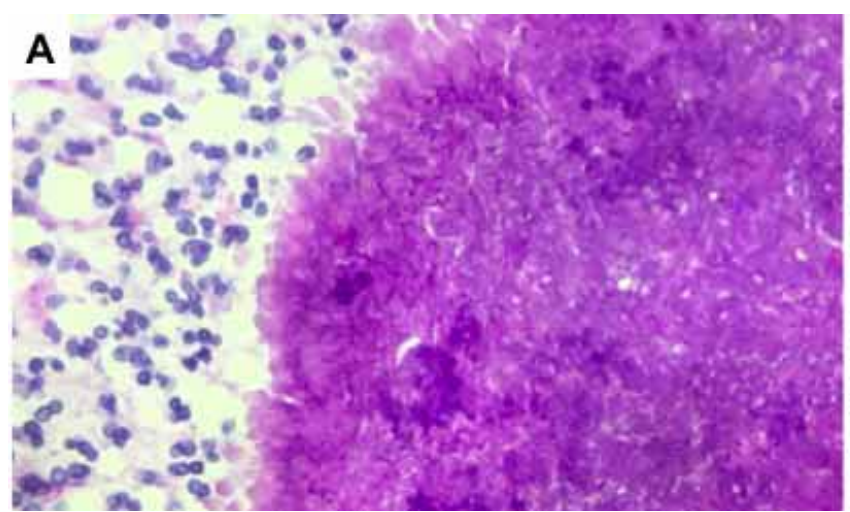

\section{DISCUSIÓN}

El diagnóstico de actinomicosis abdominal suele realizarse con el estudio histológico de los especímenes de extensas cirugías; pues el diagnóstico clínico se plantea en menos del $10 \%$ de los casos, lo que se acepta, dada la baja prevalencia de la entidad, la forma de presentación inusual y el bajo índice de sospecha. Por ello, se admite que la confirmación diagnóstica puede plantearse por la detección de una masa purulenta bacteriana, la confirmación de una masa purulenta por histología, o la positividad de un cultivo para Actinomyces spp. (Tsujimura et al.). Sin embargo, las muestra de tejido o de fluidos son fundamentales para plantear el

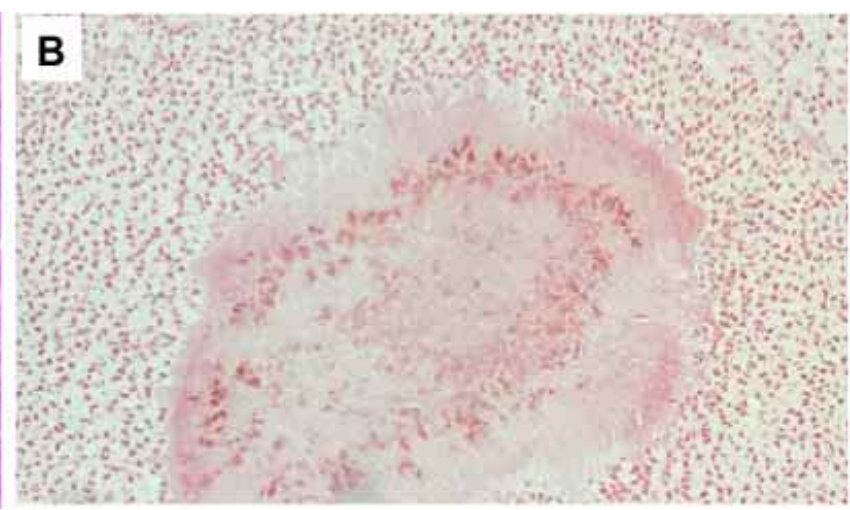

Fig. 4. Se observan elementos filamentosos positivos en tinción de PAS (A), y negativos para tinción de Grocott (B). 


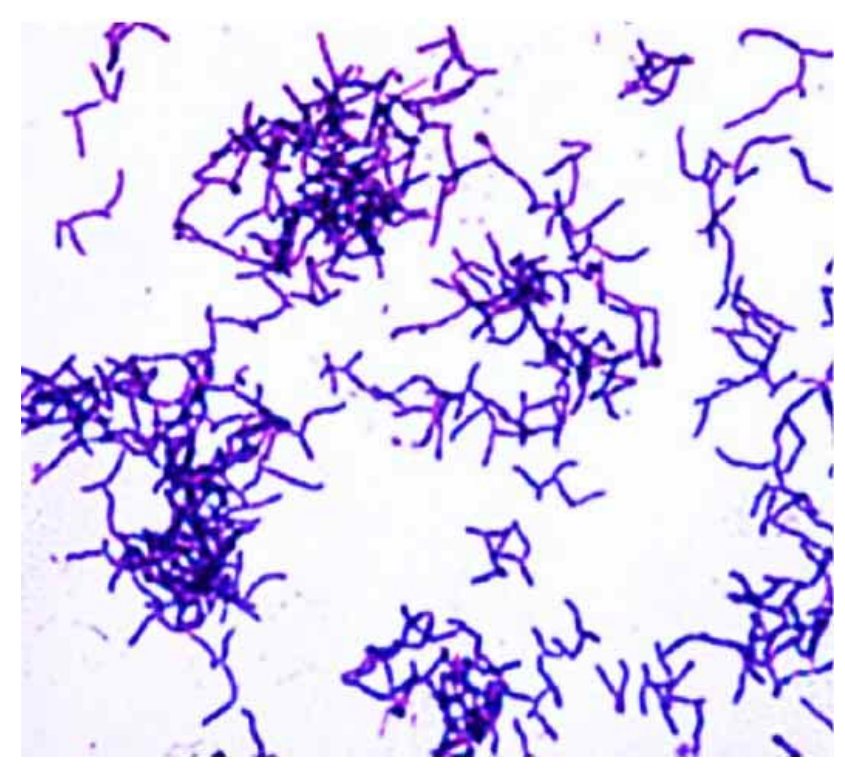

Fig. 5. Tinción de Gram. Se verifican bacilos Gram-positivos, no esporulados, característicos de Actinomyces israelii.

diagnóstico. La tinción de Gram parece ser más sensible que los cultivos (a pesar que siguen considerándose el estándar de referencia), especialmente en pacientes que han recibido antibióticos (Wong et al., 2011; Bonnefond et al., 2016). No obstante ello, el hecho más característico, es la aparición de gránulos de azufre en el examen histopatológico (García García et al.; Wong et al.; Boyanova et al.).

La actinomicosis abdominal puede desarrollarse a partir de una apendicitis, diverticulitis, perforación de víscera hueca por cuerpos extraños, así como por el uso de dispositivos intrauterinos (Tsujimura et al.).

La evidencia actual, respalda la ponencia de que en pacientes con diagnóstico confirmado, el tratamiento médico sería suficiente para tratar la infección. Para ello, la aspiración con aguja fina guiadas por imágenes, han demostrado ser eficaces en la obtención de muestras; así como para realizar un drenaje percutáneo posterior (García García et al.; Wong et al.; Boyanova et al.; Bonnefond et al.). Pero, la utilización de antibióticos aislados, no sería suficiente para el control de la enfermedad, por la dificultad del fármaco de penetrar hasta el centro de la lesión debido a que se encuentran rodeadas de gruesos tejidos conectivos y son poco vascularizadas (Wong et al.; Tsujimura et al.).

Sin embargo, las estrategias terapéuticas dependen de factores tales como localización de la lesión (tórax, miembros, cavidad abdominal, etc.) y extensión de la enfermedad (localizada o sistémica). Es evidente, que el uso de antibióticos es necesario; pero, como se trata de una entidad poco prevalente (en especial la localización abdominal); no existe evidencia de buen nivel ni guías de práctica clínica que apoyen la indicación de algún régimen sobre otro. Se sabe que Actinomyces spp. son sensibles a la penicilina; por lo que las recomendaciones incluyen un ciclo de penicilina G 20 millones de UI/día, seguido de penicilinas orales (como amoxicilina), por tiempos variables, bajo estricto control tomográfico o de resonancia nuclear. Pero, en aquellos casos de resecciones quirúrgicas terapéuticas (por indicación primaria o tratamiento enfermedad refractaria al antibiótico), no existe claridad sobre el beneficio de tratamientos antibióticos de larga duración (Wong et al.).

Al parecer el tratamiento mas efectivo para la actinomicosis abdominal es la cirugía y la ulterior administración de antimicrobianos por períodos no bien determinados, con el objetivo de reducir eventuales recidivas postoperatorias, en especial, en aquellos casos de enfermedad diseminada (Pujol Molinara et al.; García García et al.; Wong et al.; Tsujimura et al.). Además, se ha de considerar que la resección quirúrgica se realiza habitualmente, debido a la dificultad para descartar la sospecha de una neoplasia maligna (Tsujimura et al.).

En resumen, presentamos un caso de actinomicosis de pared abdominal con infiltración hepática que simulaba una neoplasia maligna en una paciente joven, cuyo diagnóstico fue confirmado por anatomía patológica tras la exéresis total de la masa, descartándose la existencia de una neoplasia.

MANTEROLA, C.; GRANDE, L. \& OTZEN, T. Abdominal wall actinomycosis with hepatic infiltration mimicking a malignant neoplasm. Case report. Int. J. Morphol., 37(3):1033-1037, 2019.

SUMMARY: Actinomyces infection (actinomycosis) may create diagnostic conflicts and be confused with malignant neoplasms, especially in the abdomen. The objective of this study was to report a case of abdominal wall actinomycosis with hepatic infiltration, and review the existing evidence. Female patient, 33 years of age, with no surgical history or use of intra-uterine devices. She consulted for abdominal pain and palpable mass at the epigastrium. It was studied with images, which allowed verifying an abdominal wall mass with hepatic fistulae. A broad extirpation of the lesion was performed. The histological study revealed sulfur granules consistent with actinomyces. The patient has evolved satisfactorily, without problems; and is currently in treatment with amoxicillin. We present an unusual case of abdominal wall actinomycosis with hepatic infiltration that resulted in a difficult diagnosis. When a large intraperitoneal mass is found, actinomycosis needs to be included as a differential diagnoses.

KEY WORDS: Actinomycosis; Actinomycosis/surgery; Aerobic actinomyces infection [Supplementary Concept]; Abdominal Neoplasms; Liver Abscess; Pyogenic; Abdominal wall mass. 


\section{REFERENCIAS BIBLIOGRÁFICAS}

Apotheloz, C. \& Regamey, C. Disseminated infection due to Actinomyces meyeri: case report and review. Clin. Infect. Dis., 22(4):621-5, 1996.

Bonnefond, S.; Catroux, M.; Melenotte, C.; Karkowski, L.; Rolland, L.; Trouillier, S. \& Raffray, L. Clinical features of actinomycosis: A retrospective, multicenter study of 28 cases of miscellaneous presentations. Medicine (Baltimore), 95(24):e3923, 2016.

Boyanova, L.; Kolarov, R.; Mateva, L.; Markovska, R. \& Mitov, I. Actinomycosis: a frequently forgotten disease. Future Microbiol., 10(4):613-28, 2015.

Deshmukh, N. \& Heaney, S. J. Actinomycosis at multiple colonic sites. Am. J. Gastroenterol., 81(12):1212-4, 1986.

Filipovic, B.; Milinic, N.; Nikolic, G. \& Ranthelovic, T. Primary actinomycosis of the anterior abdominal wall: case report and review of the literature. J. Gastroenterol. Hepatol., 20(4):517-20, 2005.

García García, J. C.; Núñez Fernández, M. J.; Cerqueiro González, J. M.; García Martín, C.; Rodríguez García, J. C.; Anibarro García, L.; de Lis Muñoz, J. M. \& Piñeiro Gómez-Durán, L. Actinomicosis primaria de la pared abdominal. Descripción de dos casos y revisión de la literatura. An. Med. Interna, 18(2):36-9, 2001

Hamid, D.; Baldauf, J. J.; Cuenin, C. \& Ritter, J. Treatment strategy for pelvic actinomycosis: case report and review of the literature. Eur. $J$. Obstet. Gynecol. Reprod. Biol., 89(2):197-200, 2000.

Kim, K. H.; Lee, J. S.; Cho, H. J.; Choi, S. B.; Cheung, D. Y.; Kim, J. I. \& Lee, I. K. A case of abdominal wall actinomycosis. Korean J. Gastroenterol., 65(4):236-40, 2015.

Manterola, C. Laparotomía en "J". Una vía de acceso alternativa para cirugía abdominal alta. Int. J. Med. Surg. Sci., 1(2):185-90, 2014.

Manterola, C.; del-Sol, M.; Ottone, N. \& Otzen, T. Radiological and surgical anatomy of the liver and fundamentals of the various options liver resections. Int. J. Morphol., 35(4):1525-39, 2017.

Meyer, P.; Nwariaku, O.; McClelland, R. N.; Gibbons, D.; Leach, F.; Sagalowsky, A. I.; Simmang, C. \& Jeyarajah, D. R. Rare presentation of actinomycosis as an abdominal mass: report of a case. Dis. Colon Rectum, 43(6):872-5, 2000.

Pujol Molinar, M.; Masferrer Sala, L. I.; Piñol Suñé, J.; Buqueras Bujosa, C.; Mata Sancho, F.; Farreras Catasús, N. \& Ortiz de Zárate, L. Actinomicosis abdominal: diagnóstico diferencial de una masa colónica. Cir. Esp., 68(2):91-184, 2000.

Tsujimura, N.; Takemoto, H.; Nakahara, Y.; Wakasugi, M.; Matsumoto, T.; Nishioka, K.; Takachi, K.; Oshima, S. \& Yoshida, K. Intraabdominal actinomycosis resulting in a difficult to diagnose intraperitoneal mass: A case report. Int. J. Surg. Case Rep., 45:101-3, 2018.

Wacharachaisurapol, N.; Bender, J. M.; Wang, L.; Bliss, D.; Ponrartana, S. \& Pannaraj, P. S. Abdominal Actinomycosis in Children: A Case Report and Literature Review. Pediatr. Infect. Dis. J., 36(3):e76-e79, 2017.

Wong, V. K.; Turmezei, T. D. \& Weston, V. C. Actinomycosis. B. M. J., 343:d6099, 2011.

Yi, F.; Prasad, S.; Sharkey, F. \& Kahlenberg, M. Actinomycotic infection of the abdominal wall mimicking a malignant neoplasm. Surg. Infect. (Larchmt.), 9(1):85-9, 2008.
Dirección para correspondencia:

Dr. Carlos Manterola Delgado

Departamento de Cirugía y CEMyQ

Universidad de La Frontera.

Manuel Montt 112, oficina 408

Temuco

CHILE

Email: carlos.manterola@ufrontera.cl

Recibido : 22-10-2018

Aceptado : 20-04-2019 